\title{
A stochastic model for bacteria-driven micro-swimmers
}

\author{
Christian Esparza López, Albane Théry and Eric Lauga
}

Received Date

Accepted Date

DOI: $10.1039 / x x x x x x x x x x$

Experiments have recently shown the feasibility of utilising bacteria as micro-scale robotic de-

www.rsc.org/journalname vices, with special attention paid to the development of bacteria-driven micro-swimmers taking advantage of built-in actuation and sensing mechanisms of cells. Here we propose a stochastic fluid dynamic model to describe analytically and computationally the dynamics of microscopic particles driven by the motion of surface-attached bacteria undergoing run-and-tumble motion. We compute analytical expressions for the rotational diffusion coefficient, the swimming speed and the effective diffusion coefficient. At short times, the mean squared displacement (MSD) is proportional to the square of the swimming speed, which is independent of the particle size (for fixed density of attached bacteria) and scales linearly with the number of attached bacteria; in contrast, at long times the MSD scales quadratically with the size of the swimmer and is independent of the number of bacteria. We then extend our result to the situation where the surface-attached bacteria undergo chemotaxis within the linear response regime. We demonstrate that bacteria-driven particles are capable of performing artificial chemotaxis, with a chemotactic drift velocity linear in the chemical concentration gradient and independent of the size of the particle. Our results are validated against numerical simulations in the Brownian dynamics limit and will be relevant to the optimal design of micro-swimmers for biomedical applications.

\section{Introduction}

Miniaturisation of actuators and efficient power sources are two of the biggest technical challenges in the design and fabrication of microscopic robots ${ }^{12}$. As is often the case, Nature can offer insight into overcoming these challenges. Flagellated bacteria, such as the well-studied E. coli, are known to be efficient swimmers with intricate sensing capabilities ${ }^{33}$ and they have inspired scientists to integrate living cells and synthetic components into bio-hybrid devices 214:12. Bacteria-driven micro-swimmers have received special attention due to their potential applications in medicine such as targeted drug delivery 8113,19 . Commonly, a micro-swimmer consists of a synthetic substrate such as a micro-plate ${ }^{720}$ or a micro-bead ${ }^{[16] 1721}$ with just a few or many bacteria attached to their surfaces (see experimental example in Fig. 17). The attachment of the cells to the surface is often random but can be controlled by patterning the substrate using chemical or physical techniques 5 522. The dynamics of a passive bead driven by surface-attached bacteria is a result of the precise behaviour of each cell. When free swimming, a peritrichous bacterium such as $E$. coli moves in a series of nearly straight paths

Department of Applied Mathematics and Theoretical Physics, University of Cambridge, Cambridge CB3 OWA, United Kingdom.

$\ddagger$ e.lauga@damtp.cam.ac.uk ('runs'), interrupted by quick changes in orientation ('tumbles'). This so-called run-and-tumble motion consists therefore of two stages: (i) the running phase, in which all the helical flagellar filaments rotate counterclockwise (when measured from behind the cell) and form a bundle which aligns with the cell body and whose rotation in the fluid propels the cell forward; (ii) the tumble phase, in which at least one flagellar filament rotates in the clockwise direction and leaves the bundle resulting in a reorientation of the whole cell ${ }^{[23}$. During the running phase, the bacterium experiences a constant propulsive force and torque along the same direction. Both, force and torque, balance with the corresponding hydrodynamic translational and rotational drags, so that the cell is overall force- and torque-free. In contrast, in the tumbling phase the bacterium experiences no propulsive force but a torque which reorients the cell body $\underline{15}$. The running and tumbling events for E. coli are known to be Poisson distributed ${ }^{25}$ with each run lasting for approximately one second while tumbles last just a tenth of a second 23 . These times can be altered by chemical concentration gradients in the environment surrounding the cell. Bacteria such as E. coli can measure temporal differences in concentration of certain chemicals during their locomotion, for example aspartate, and reduce their tumbling rate if moving up the gradient 2627 . As a result, the trajectory of a swimming bacterium is an isotropic random walk in homogeneous environments and 

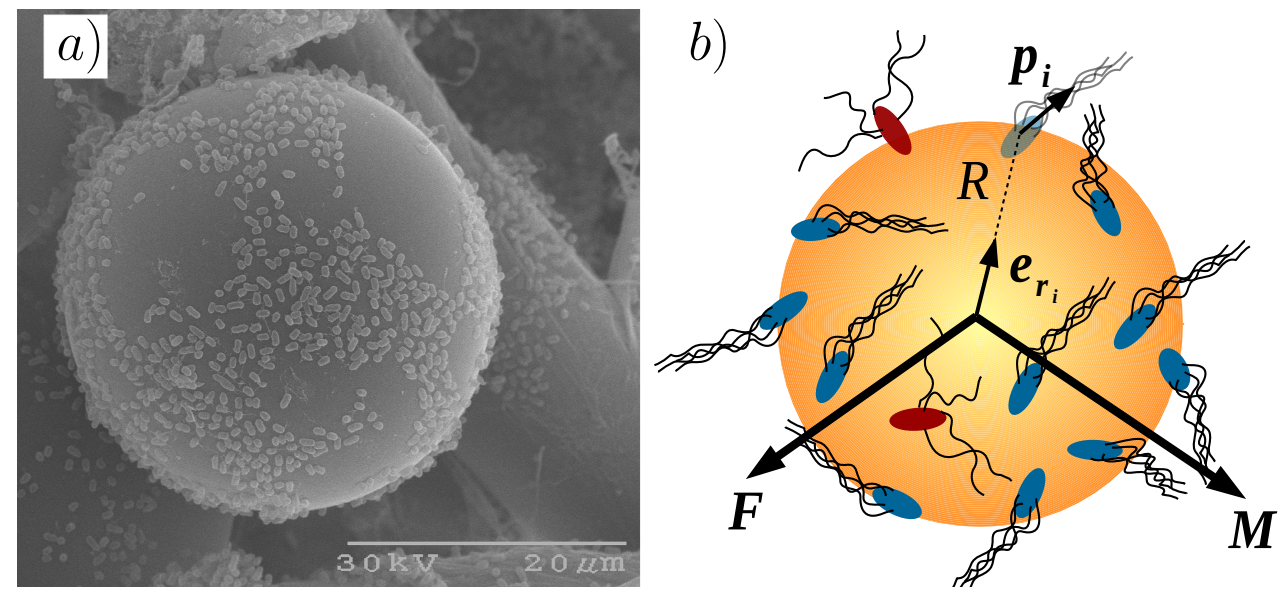

Fig. 1 Bacteria-driven micro-swimmer. (a) Scanning electron microscope (SEM) image of a $30 \mu \mathrm{m}$ diameter bead with surface-attached bacteria. The individual cells are seen as small dots on the smooth surface of the bead (reprinted by permission from Kim et al. ${ }^{24}$. Copyright 2012 from Springer Nature). (b) Schematic representation of a bacteria-driven particle of radius $R$. The unit vectors $\mathbf{e}_{r_{i}}$ and $\mathbf{p}_{\mathbf{i}}$ define the position and orientation of the $i$-th bacterium. The total hydrodynamic force, $\mathbf{F}$, and torque, $\mathbf{M}$, are the sum of the applied forces and torques by each bacterium, $-f \mathbf{p}_{i}$ and $-R f\left(\mathbf{e}_{r_{i}} \times \mathbf{p}_{i}\right)$ respectively.

a biased random walk when in the presence of a chemical gradient (if the cell is sensitive to it). The bias in the direction of motion is known as chemotaxis and when displayed by bacteria attached to passive particles it can potentially provide chemotactic abilities to the particles themselves 1328 . Indeed, there are many other taxis techniques which can be used to control the trajectories of synthetic micro-swimmers, such as phototaxis ${ }^{29}$ and magnetotaxis 30 . However, for medical applications chemotaxis appears to be the most natural, as not only it does not require the use of external force fields but can also be linked to the ubiquitous presence of chemical gradients in the human body.

Helical trajectories of particles with surface-attached bacteria have been observed in experiments with small beads on which only one or two bacteria can be attached ${ }^{15 / 31}$. This suggests that the cells, despite being attached to a surface, still follow a run-and-tumble dynamics, and each bacterium applies a constant force and constant torque to the bead. Following this observation, a number of mathematical models have been proposed to describe the motion of bacteria-driven micro-swimmers 714 115. The swimmers are modelled as spherical particles actuated by random forces and torques taken as the sums of the individual propulsive forces and torques applied by each bacterium. Given the small velocity scales, the motion occurs at low Reynolds number and is governed by Stokes laws which relate linearly the applied forces and torques to the linear and angular velocities of the bead. Although these models have been validated numerically against experimental results $813-16$, very few analytical expressions have been derived. For example, it is known that for large number of surface-attached bacteria $N$, the swimming speed increases as $\sqrt{N}[14 \mid 16$ but the dependence on the size of the micro-swimmer is unclear.

A detailed mathematical analysis of the motility properties of the micro-swimmers is essential for their optimal design and fabrication. In this study, we thus develop a stochastic model for biohybrid spherical micro-swimmers with $E$. coli bacteria attached to their surface. We derive analytical expressions for the rotational diffusion coefficient and the mean squared displacement (MSD), based on the following assumptions: (i) low-Reynolds number flow, (ii) a large number of uniformly attached bacteria, (iii) negligible thermal noise compared to the random activity of each cell and (iv) run-and-tumble dynamics for each bacterium. We first consider the situation where the chemical environment is homogenous and next investigate the effects of external chemical gradients, assuming a linear response of each bacterium. In particular, we show rigorously that the individual capabilities of E. coli cells to move up concentration gradients are inherited by the micro-swimmers on which they are attached. Our analytical results are validated against numerical simulations (using a standard Brownian dynamics framework) and past experimental results ${ }^{813}-16$.

\section{Mathematical model and numerical simu- lations}

\subsection{Micro-swimmers in homogeneous environments}

Following previous studies ${ }^{14115}$, we model the micro-swimmers as passive spherical particles of radius $R$ with a number $N$ of uniformly distributed bacteria attached to their surface. Reported densities of attachment in experiments range from one bacterium per $12 \mu \mathrm{m}^{2}$ up to one per $7 \mu \mathrm{m}^{2} 16$. The bacteria are assumed to be fixed in position and orientation with respect to the surface of the particle. This condition is satisfied in practice by using strong chemical binding such as streptavidin-biotin interactions ${ }^{15}$. Previous numerical investigations suggest that the direction of the flagellar bundle is unaffected by the fluid flow in the vicinity of the swimmer ${ }^{14}$, therefore the flagellar bundle is assumed to align with the orientation of the cell body with no change as the swimmer moves. Each cell is assumed to perform its own, independent run-and-tumble dynamics. The reaction torques in the running and tumbling states have magnitudes $\left|\mathbf{M}_{R}\right| \simeq 0.7 \mathrm{pN} \mu \mathrm{m}$ and $\left|\mathbf{M}_{T}\right| \simeq 0.4 \mathrm{pN} \mu \mathrm{m}$ respectively 15 . These 


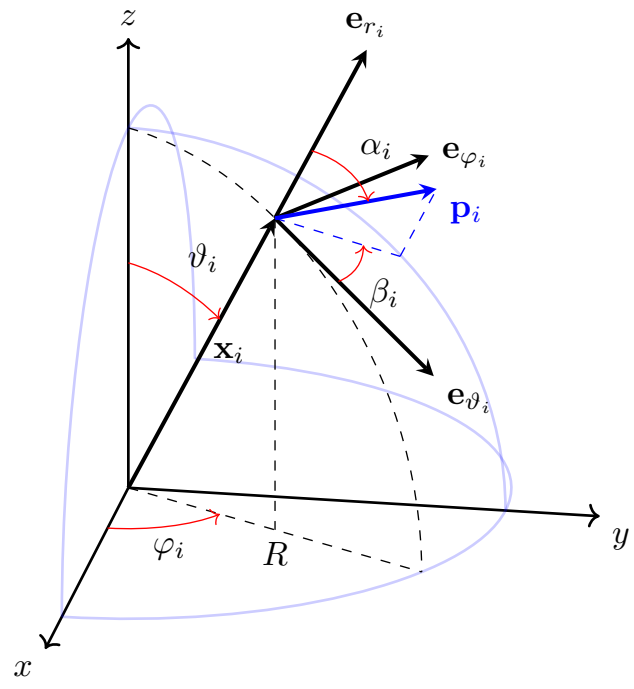

Fig. 2 Notation for the location and orientation of each bacterium on the surface of the particle. The $i$-th bacterium is located at $\mathbf{x}_{i}=R \mathbf{e}_{r_{i}}$ where $R$ is the radius of the particle and $\mathbf{e}_{r_{i}}$ is the radial unit vector defined by the polar and azimuth angles $\vartheta_{i}$ and $\varphi_{i}$ with respect to the body frame $\{x, y, z\}$. The orientation of the bacterium relative to the bead surface is given by the unit vector $\mathbf{p}_{i}$, which is the radial unit vector defined by the polar and azimuth angles $\alpha_{i}$ and $\beta_{i}$ with respect to the local spherical coordinate system $\left\{\mathbf{e}_{\vartheta_{i}}, \mathbf{e}_{\varphi_{i}}, \mathbf{e}_{r_{i}}\right\}$. Each bacterium is assumed to push on the fluid along $\mathbf{p}_{i}$ and thus to exert a force on the particle along $-\mathbf{p}_{i}$.

torques can be neglected if we focus on sufficiently large particles such that $\left|\mathbf{M}_{R, T}\right| \sqrt{\left\langle\sin ^{2} \alpha\right\rangle_{\alpha}} \ll f R$, where $f \sim 0.3-0.48 \mathrm{pN}$ is the average propulsive force exerted by each bacterium ${ }^{1516}$ and the angle $\alpha$ defined in Fig. 2 denotes the orientation of the bacterium flagella bundle with respect to the radial direction. In other words, for large particles, the reorientation of the swimmer is dominated by the moment-arm torque induced by the propulsive forces of the cells. Finally, thermal noise can also be neglected as it induces typical forces three orders of magnitude smaller than the propulsive forces from the bacteria. We model therefore each bacterium as a two-state machine which exerts a force of magnitude $f$ when running and no force when tumbling. The transition between the running and the tumbling states is modelled as a continuous time Markov chain with transition rates $\lambda_{R}$ and $\lambda_{T}$ from the tumbling to the running state and vice-versa, respectively (see Fig. 3). The values for $E$. coli in homogeneous environments (no chemical gradients) are $\lambda_{R} \simeq 10 \mathrm{~s}^{-1}$ and $\lambda_{T} \simeq 10 / 9 \mathrm{~s}^{-1}$. Furthermore, the swimming motion occurs at low Reynolds number $R e \simeq 10^{-4}[25$. The linear and angular velocities of the microswimmer, denoted by $\mathbf{V}$ and $\omega$, are obtained by force and moment balance using Stokes law

$$
\begin{aligned}
& \mathbf{F}=6 \pi \mu R \mathbf{V}=-f \sum_{i=1}^{N} \varepsilon_{i} \mathbf{p}_{i}, \\
& \mathbf{M}=8 \pi \mu R^{3} \omega=-f R \sum_{i=1}^{N} \varepsilon_{i} \mathbf{e}_{r_{i}} \times \mathbf{p}_{i},
\end{aligned}
$$

where $\mu$ is the dynamic viscosity of the fluid, $\mathbf{F}$ and $\mathbf{M}$ are the total force and torque applied by the surface-attached cells, while

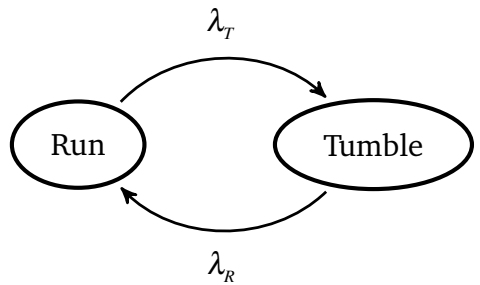

Fig. 3 The run-and-tumble motility pattern as a two-state Markov chain with transition rates $\lambda_{R}$ and $\lambda_{T}$ from the tumbling to the running state and vice-versa, respectively. Typical values of these rates for $E$. coli in homogeneous environments (no chemical gradients) are $\lambda_{T}^{-1}=0.9 \mathrm{~s}$ and $\lambda_{R}^{-1}=0.1 \mathrm{~s} 25$.

$\mathbf{e}_{r_{i}}$ and $\mathbf{p}_{i}$ are unit vectors which determine the position and orientation of the $i$-th bacterium (see Figs. 1 b and 2). Specifically, each bacterium is assumed to push on the fluid along the direction $\mathbf{p}_{i}$ of its bundle of flagellar filaments and thus to exert a force on the particle along $-\mathbf{p}_{i}$. In Eqs. (1) and (2), the stochastic variable $\varepsilon_{i}$ determines the state of the $i$-th bacterium with $\varepsilon_{i}=1$ when running and $\varepsilon_{i}=0$ when tumbling. The position and orientation of the micro-swimmer evolve in time according to

$$
\begin{array}{r}
\dot{\mathbf{X}}=\mathbf{V}, \\
\dot{\mathbf{n}}=\omega \times \mathbf{n},
\end{array}
$$

where the dot denotes a derivative with respect to time and $\mathbf{n}$ is a body-fixed unit vector. We integrate Eq. (3) numerically using an Euler method and Eq. (4) using the mid point method presented in Refs. $\frac{32 \sqrt{33}}{3}$ with a time step $\mathrm{d} t=0.01 \mathrm{~s}$. We consider $10^{4}$ different configurations, sampling the geometrical angles $\vartheta_{i}, \varphi_{i}, \alpha_{i}$ and $\beta_{i}$, which describe the location and orientation of the cells on the bead surface (see Fig. 2), from uniform distributions in the intervals $[0, \pi],[0,2 \pi),\left[\alpha_{\max }, \alpha_{\min }\right]$ and $[0,2 \pi)$ respectively. The maximum and minimum deviation angles from the radial direction are $\alpha_{\min }=30^{\circ}$ and $\alpha_{\max }=85^{\circ}$ following Ref. ${ }^{14}$.

\subsection{Run-and-tumble dynamics}

If we denote by $p_{R}(t)$ and $p_{T}(t)$ the probabilities of finding a particular bacterium in the running and the tumbling states respectively, the master equation for the run-and-tumble dynamics is given by

$$
\left[\begin{array}{c}
\dot{p}_{R} \\
\dot{p}_{T}
\end{array}\right]=\left[\begin{array}{cc}
-\lambda_{T} & \lambda_{R} \\
\lambda_{T} & -\lambda_{R}
\end{array}\right]\left[\begin{array}{c}
p_{R} \\
p_{T}
\end{array}\right]
$$

where $\lambda_{R}$ and $\lambda_{T}$ are the transitions rates defined earlier. The system in Eq. (5) can be easily integrated by considering the normalisation condition $p_{R}+p_{T}=1$, with the result

$$
\left[\begin{array}{c}
p_{R} \\
p_{T}
\end{array}\right]=\left[\begin{array}{c}
\frac{\lambda_{R}}{\lambda_{R}+\lambda_{T}}+A e^{-\left(\lambda_{R}+\lambda_{T}\right) t} \\
\frac{\lambda_{T}}{\lambda_{T}+\lambda_{R}}-A e^{-\left(\lambda_{R}+\lambda_{T}\right) t}
\end{array}\right],
$$

where $A$ is a constant determined by the initial state of the bacterium. In particular, if the bacterium is running at time $t=0$ we 
have $p_{R}(0)=1$, hence

$$
\left[\begin{array}{c}
p_{R R} \\
p_{T R}
\end{array}\right]=\left[\begin{array}{c}
\frac{\lambda_{R}+\lambda_{T} e^{-\left(\lambda_{R}+\lambda_{T}\right) t}}{\lambda_{R}+\lambda_{T}} \\
\frac{\lambda_{T}-\lambda_{T} e^{-\left(\lambda_{R}+\lambda_{T}\right) t}}{\lambda_{R}+\lambda_{T}}
\end{array}\right]
$$

where we denote by $p_{R R}(t), p_{T R}(t)$ the probability for a bacterium to be running or tumbling at time $t$ given that it was running at time $t=0$. Furthermore, in the steady state, the results of Eq. (6) reduce to

$$
\left[\begin{array}{l}
p_{R}^{s} \\
p_{T}^{s}
\end{array}\right]=\left[\begin{array}{c}
\frac{\lambda_{R}}{\lambda_{R}+\lambda_{T}} \\
\frac{\lambda_{T}}{\lambda_{T}+\lambda_{R}}
\end{array}\right] .
$$

In our numerical simulations, we assume that we have waited long enough so that the system has reached steady state. We therefore determine the state of the variables $\varepsilon_{i}$ by sampling a pseudo random number $r_{i}$ from a uniform distribution and comparing it with $p_{R}^{s}$ for the first step and $p_{R R}(\mathrm{~d} t), p_{R T}(\mathrm{~d} t)$ for the following steps. Here $p_{R T}$ is obtained from Eq. (6) by taking $p_{R}(0)=0$. In the steady state, the mean of the variables $\varepsilon_{i}(t)$ is

$$
\left\langle\varepsilon_{i}(t)\right\rangle=P\left(\varepsilon_{i}(t)=1\right)=p_{R}^{s}=\frac{\lambda_{R}}{\lambda_{R}+\lambda_{T}},
$$

while the time autocorrelation is given by

$$
\begin{aligned}
\left\langle\varepsilon_{i}(t) \varepsilon_{j}(s)\right\rangle & =P\left(\varepsilon_{i}(t)=1, \varepsilon_{j}(s)=1\right) \\
& =P\left(\varepsilon_{j}(s)=1 \mid \varepsilon_{i}(t)=1\right) p_{R}^{s} .
\end{aligned}
$$

If the bacteria behave independently from each other, the probability of the $j$-th bacterium being running at time $s$, given that the $i$-th bacterium is also running at time $t$, is $p_{R R}(|s-t|)$ for $i=j$ and $p_{R}^{s}$ otherwise. Hence

$$
\left\langle\varepsilon_{i}(t) \varepsilon_{j}(s)\right\rangle=\left(p_{R}^{s}\right)^{2}\left(1+\delta_{i j} \frac{\lambda_{T}}{\lambda_{R}} e^{-\left(\lambda_{R}+\lambda_{T}\right)|s-t|}\right) .
$$

We will use Eqs. (9) and (11) to evaluate the MSD bellow.

\subsection{Chemotaxis of a single bacterium}

Bacteria such as E. coli are able to navigate through chemical gradients by using an inhibition of the mechanism that inverts the polarity of their bacterial motor when the cell swims in a favourable direction 3 . Polarity inversion is responsible for tumbling events, and therefore runs are extended when swimming up (resp. down) the chemoattractant (resp. repellent) gradient 34 . To investigate whether the individual chemotaxis of single cells contributes collectively to chemotaxis of the micro-swimmer, we use the simplest mathematical model that describes chemotaxis of a single bacterium. Specifically, we model each organism as equipped with an internal sensor that responds to temporal variations in the chemical concentration of its environment by modifying the tumbling rate, $\lambda_{T}$, i.e. the expected rate at which running cells stop running and transition to a tumble 27 . For weak chemical concentrations $c(t)$ sensed by the bacterium, the variation in $\lambda_{T}$ is captured by a linear response as 35

$$
\lambda_{T}(t)=\lambda_{0}\left(1-\int_{-\infty}^{t} K\left(t-t^{\prime}\right) c\left(t^{\prime}\right) \mathrm{d} t^{\prime}\right),
$$

where $\lambda_{0}$ is the mean tumbling rate when $c=c_{0}$ is constant. The function $K(t)$ is a memory kernel which describes the response and has been measured for $E$. coli by Segall et al. 26 . For a constant concentration gradient, the variation in the concentration experienced by the bacterium will depend on its position as follows

$$
c(t)=c_{0}+\mathbf{r}(t) \cdot \nabla c=c_{0}+\nabla c \cdot \int_{0}^{t} \mathbf{v}\left(t^{\prime}\right) \mathrm{d} t^{\prime}
$$

where $c_{0}$ is a constant background concentration and $\mathbf{r}(t)$ and $\mathbf{v}(t)$ are, respectively, the position and velocity of the bacterium at time $t$ measured from the beginning of the last running event. The memory kernel $K(t)$ satisfies the condition of adaptability 27

$$
\int_{0}^{\infty} K(t) \mathrm{d} t=0
$$

which ensures $\lambda_{T}(t)=\lambda_{0}$ when $c$ is kept constant. Inserting Eqs. (13) into Eq. (12) we obtain the tumbling rate as

$$
\lambda_{T}(t)=\lambda_{0}\left[1-\nabla c \cdot \int_{0}^{\infty} \int_{0}^{t-s} K(s) \mathbf{v}\left(s^{\prime}\right) \mathrm{d} s^{\prime} \mathrm{d} s\right] .
$$

For computational convenience, we consider in our numerical simulations the simplest possible adaptive kernel. Specifically, we take only two impulses localised at $t_{1}$ and $t_{2}$ with $t_{1}<t_{2}$, and therefore write $K(t)=\kappa\left[\delta\left(t-t_{1}\right)-\delta\left(t-t_{2}\right)\right]$, where $\kappa$ is the magnitude of the response. This models a cell which compares concentrations at two different, specific times in the past, and changes its tumbling rate accordingly. Substitution of this kernel into Eq. (15) yields

$$
\lambda_{T}(t)=\lambda_{0}\left\{1-\kappa \nabla c \cdot\left[\mathbf{r}\left(t-t_{1}\right)-\mathbf{r}\left(t-t_{2}\right)\right]\right\} .
$$

Note that, since any function can be expressed as a superposition of impulses, it is possible to treat more general chemotactic responses (such as those proposed by Clark et al. $\$ 36$ or Celani et al.37) in a similar manner by approximating $K(t)$ by a sum of delta functions, $K(t) \simeq \sum \kappa_{j} \delta\left(t-t_{j}\right)$, where $t_{j}$ 's are delay times and where the $\kappa_{j}$ 's determine the intensity of the response subject to $\sum \kappa_{j}=0$.

\subsection{Chemotaxis of bacteria-driven micro-swimmers}

Chemotaxis is included numerically in our model by modifying the tumbling rate of each bacterium according to Eq. (16). We make the simplifying assumption that the particle is permeable to the chemical so that the chemical gradient is not perturbed by its presence. In general, we should evaluate $\mathbf{r}(t)$ in Eq. (16) as the position of each cell. However, if we consider shallow gradients such that $\kappa|\nabla c| R \ll 1$, then it is appropriate to neglect variations in concentration along the surface of the particle and substitute the position of the centre of the micro-swimmer for $\mathbf{r}(t)$ in Eq. (16).

We run the simulation from the previous sections for an 


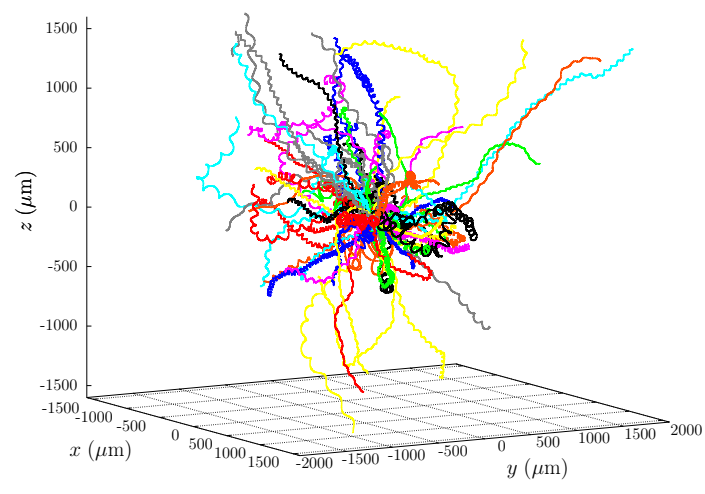

Fig. 4 Typical trajectories of bacteria-driven micro-swimmers of radius $R=10 \mu \mathrm{m}$ with bacteria density $\rho=1 / 12 \mu \mathrm{m}^{-2}$, individual propulsive forces $f=0.48 \mathrm{pN}$, and transition rates $\lambda_{T}^{-1}=0.9 \mathrm{~s}$ and $\lambda_{R}^{-1}=0.1 \mathrm{~s}$. The simulation time is $t=5 /\left(3 D_{r}\right) \simeq 224 \mathrm{~s}$, where $D_{r}$ is given by Eq. (39). One hundred different realisations are shown.

ensemble of $10^{4}$ beads, now evaluating the tumbling rate at each step using Eq. (16) with $\mathbf{r}(t)$ taken to be the position of the centre of the bead. We further take the position of the two impulses in the kernel to coincide with the maximum and minimum of the response kernel measured by Segall et al. 26 , i.e. $t_{1} \simeq 1 \mathrm{~s}$ and $t_{2} \simeq 3 \mathrm{~s}$. The intensity of the response is taken as $\kappa=|K|_{\max } \times 1 \mathrm{~s} \simeq 0.3 \mu \mathrm{M}^{-1} 37$. As we require the second term in brackets in Eq. (16) to remain small and $\mathbf{r}(t) \sim U_{e}\left(t_{2}-t_{1}\right)$, where $U_{e} \sim 10 \mu \mathrm{m} / \mathrm{s}$ is the swimming speed of the micro-swimmers 16 , we choose $\kappa|\nabla c| \sim 10^{-3}-10^{-2} \mu \mathrm{m}^{-1}$, which corresponds to concentration gradients $|\nabla c| \sim 3 \times 10^{-3}-3 \times 10^{-2} \mathrm{mM} / \mathrm{mm}$, so that $\kappa|\nabla c| R \sim 10^{-2}-10^{-1}$ and the tumbling rate is reduced by approximately one tenth. The chemical gradient is set up along the $z$ direction.

\section{Theoretical and numerical results}

We start this section with the description of the three-dimensional trajectories of the micro-swimmers as obtained numerically and we observe that for long time-scales these become threedimensional random walks. We then introduce a coarse-grained model to describe the diffusive behaviour of the micro-swimmers. We define the rotational diffusion coefficient, $D_{r}$, the effective speed, $U_{e}$, the effective diffusion coefficient, $D_{e}$ and derive analytical expressions for each one of them. Next, we show that in the presence of a non uniform chemical concentration field, to which the bacteria respond chemotactically, the micro-swimmers perform a biased random walk and we quantify their response in terms of a drift speed $v_{d}$, for which we derive an analytical expression. Throughout this section, we validate the analytical results against our numerical simulations.

\subsection{Three-dimensional trajectories of bacteria-driven micro-swimmers}

We present first the results in isotropic environments, i.e. constant concentration field and thus constant tumbling rate. Typical trajectories for the centre of the particle, resulting from numerical integration of equations (3) and (4), are shown in Fig. 4. At short time scales, the applied force and torque are nearly constant and the resulting trajectories are noisy helices. For a given distribution of attached bacteria, the average force and torque acting on the particles in the steady state are given by

$$
\begin{aligned}
\langle\mathbf{F}\rangle_{\varepsilon} & =-f \sum_{i=1}^{N}\left\langle\varepsilon_{i}\right\rangle_{\varepsilon} \mathbf{p}_{i}=-f p_{R}^{s} \sum_{i=1}^{N} \mathbf{p}_{i} \\
\langle\mathbf{M}\rangle_{\varepsilon} & =-f R \sum_{i=1}^{N}\left\langle\varepsilon_{i}\right\rangle_{\varepsilon}\left(\mathbf{e}_{r_{i}} \times \mathbf{p}_{i}\right)=-f R p_{R}^{s} \sum_{i=1}^{N}\left(\mathbf{e}_{r_{i}} \times \mathbf{p}_{i}\right),
\end{aligned}
$$

where $\langle\cdot\rangle_{\varepsilon}$ denotes the average over the probability distribution Eq. (6). Defining the vectors $\mathbf{a} \equiv \sum \mathbf{p}_{i}$ and $\mathbf{b} \equiv \sum\left(\mathbf{e}_{r_{i}} \times \mathbf{p}_{i}\right)$, we can express the average radius and pitch of the trajectories as follows

$$
\begin{aligned}
& \mathscr{R}=\frac{4}{3} R^{2} \frac{\left|\langle\mathbf{F}\rangle_{\varepsilon} \times\langle\mathbf{M}\rangle_{\varepsilon}\right|}{\left|\langle\mathbf{M}\rangle_{\varepsilon}\right|^{2}}=\frac{4}{3} R \frac{|\mathbf{a} \times \mathbf{b}|}{|\mathbf{b}|^{2}} \\
& \mathscr{P}=\frac{8 \pi}{3} R^{2} \frac{\left|\langle\mathbf{F}\rangle_{\varepsilon} \cdot\langle\mathbf{M}\rangle_{\varepsilon}\right|}{\left|\langle\mathbf{M}\rangle_{\varepsilon}\right|^{2}}=\frac{8 \pi}{3} R \frac{|\mathbf{a} \cdot \mathbf{b}|}{|\mathbf{b}|^{2}}
\end{aligned}
$$

In contrast, at longer times the trajectories become threedimensional random walks and their diffusive behaviour determines the motility properties of the micro-swimmers. It is well known in the theory of Brownian motion that for a random walk governed by rotational diffusion, the MSD is given by 38

$$
\left\langle r^{2}(t)\right\rangle=2 \tau_{r}^{2} U_{e}^{2}\left(\frac{t}{\tau_{r}}+e^{-t / \tau_{r}}-1\right),
$$

where the angle brackets $\langle\cdot\rangle$ denote ensemble average, $U_{e}$ is the effective speed at which the bead moves and $\tau_{r}$ is the orientation correlation time, which is the time scale of decay for the orientation correlation function, $\langle\mathbf{n}(0) \cdot \mathbf{n}(t)\rangle$. In other words, $\tau_{r}$ is the time it takes the micro-swimmer to forget its initial orientation. For short time scales, that is $t \ll \tau_{r}$, Eq. (21) reduces to $\left\langle r^{2}(t)\right\rangle=U_{e}^{2} t^{2}$, which represents ballistic motion. On the other hand, for time scales such that $\tau_{r} \ll t$ the MSD is linear in $t$, a dependence typical in diffusion processes. The constant of proportionality is the effective diffusion coefficient which is defined in three dimensions by

$$
6 D_{e} \equiv \lim _{t \rightarrow \infty} \frac{\left\langle r^{2}(t)\right\rangle}{t}=2 U_{e}^{2} \tau_{r} .
$$

Since $D_{e}$ is a macroscopic property of the micro-swimmers, it is likely that a simplified description of the trajectories ignoring the microscopic details would still lead to the same result. Furthermore, ignoring the fine structure of the driving mechanism will render the chemotaxis analysis more tractable (see Sec. 3.3). Inspired by this we propose a coarse grained model as described in the next section. We will show that the micro-swimmers MSD is described by Eq. (21) and we will derive analytical expressions for $U_{e}, \tau_{r}$ and $D_{e}$.

\subsection{Coarse-grained modelling}

We start by observing that the trajectories of the micro-swimmers such as those illustrated in Fig. 4 and Fig. 5a consist of almost undisturbed helical paths interrupted by sudden changes in direction. We thus construct a coarse-grained model replacing every 

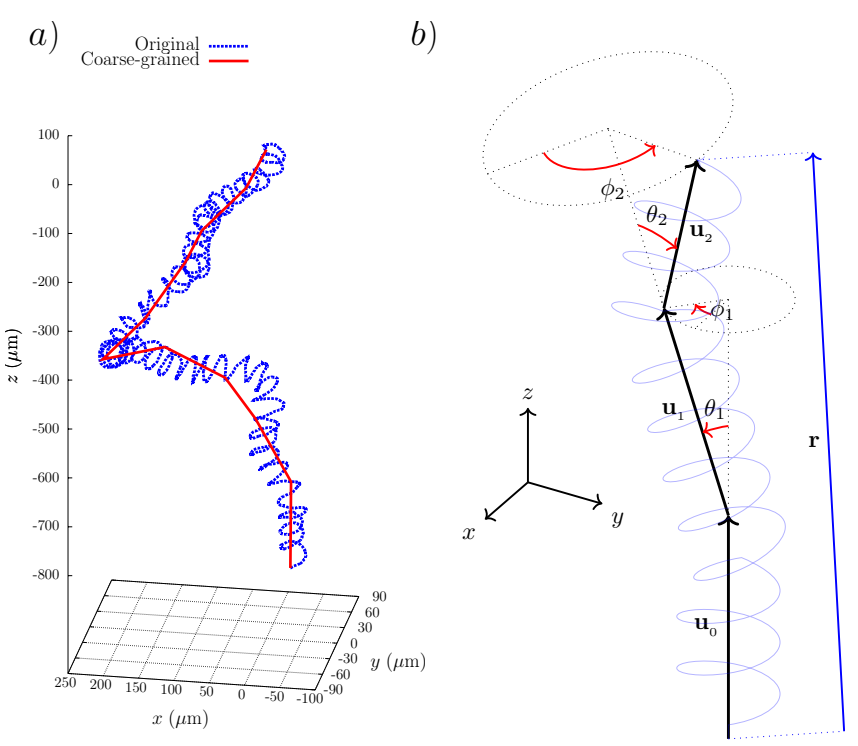

Fig. 5 Coarse graining the trajectories of particles. (a) Typical trajectory of a bacteria-driven micro-swimmer with parameters: radius $R=$ $10 \mu \mathrm{m}$, cell density $\rho=1 / 12 \mu \mathrm{m}^{-2}$, propulsive force $f=0.48 \mathrm{pN}$, and transition rates $\lambda_{T}^{-1}=0.9 \mathrm{~s}$ and $\lambda_{R}^{-1}=0.1 \mathrm{~s}$. The simulation time is $t=5 /\left(3 D_{r}\right) \simeq 224 \mathrm{~s}$, where $D_{r}$ is given by Eq. (39). The path of the swimmer is plotted in dotted blue line and can be approximated by a coarse-grained trajectory in the form of a random walk (solid red line). (b) The coarse-grained trajectory is analogous to a freely rotating chain with equal-length links. The angle between two consecutive paths $\hat{\mathbf{u}}_{i-1}$ and $\hat{\mathbf{u}}_{i}$ is denoted by $\theta_{i}$ and the internal angle of rotation, denoted by $\phi_{i}$, is the angle between the planes generated by $\left\{\hat{\mathbf{u}}_{i-2}, \hat{\mathbf{u}}_{i-1}\right\}$ and $\left\{\hat{\mathbf{u}}_{i-1}, \hat{\mathbf{u}}_{i}\right\}$.

helical trajectory by a straight path along an average axis denoted by $\hat{\mathbf{u}}_{k}$ for the $k$ th path (see Fig. 5 b). Each straight path is travelled at a constant speed $U_{e}$ for an average time $T$. The resulting trajectory is therefore a chain of equal-length links. The polar and azimuthal angles between consecutive paths $\hat{\mathbf{u}}_{i-1}$ and $\hat{\mathbf{u}}_{i}$ are denoted by $\theta_{i}$ and $\phi_{i}$ respectively. We further take $\phi_{i}$ as uniformly distributed, due to homogeneity of the space, but we will assume that there is some persistence in the direction of motion, as can be observed in Fig. 4

In polymer physics, a mathematically-identical setup is used in the freely-rotating chain model of a polymer and it is thus a textbook result that the velocity correlation function satisfies 39

$$
\begin{aligned}
\mathscr{C}(t) & \equiv\langle\mathbf{u}(0) \cdot \mathbf{u}(t)\rangle=U_{e}^{2} \sum_{q=0}^{\infty} P_{q}\left\langle\hat{\mathbf{u}}_{0} \cdot \hat{\mathbf{u}}_{q}\right\rangle_{\theta, \phi} \\
& =U_{e}^{2} \sum_{q=0}^{\infty} P_{q}\langle\cos \theta\rangle_{\theta}^{q},
\end{aligned}
$$

where $\langle\cos \theta\rangle_{\theta}$ is the average cosine of the angle between consecutive paths. Since a change in direction on a trajectory is the result of several consecutive tumbling events of the bacteria, which are Poisson distributed, the probability $P_{q}$ of observing $q$ turning events in an interval of time $t$ must be Poisson distributed as well. Considering this and the fact that the turning rate is $T^{-1}$ we ob- tain

$$
\begin{aligned}
\mathscr{C}(t) & =U_{e}^{2} \sum_{q=0}^{\infty} \frac{(t / T)^{q}}{q !} e^{-t / T}\langle\cos \theta\rangle_{\theta}^{q} \\
& =U_{e}^{2} \exp \left(-\frac{1-\langle\cos \theta\rangle_{\theta}}{T} t\right) .
\end{aligned}
$$

The MSD follows from a double integration in time of Eq. (24), leading to the result

$$
\begin{aligned}
\left\langle r^{2}(t)\right\rangle=\frac{2 U_{e}^{2} T^{2}}{\left(1-\langle\cos \theta\rangle_{\theta}\right)^{2}} & \left(\frac{1-\langle\cos \theta\rangle_{\theta}}{T} t\right. \\
& \left.+\exp \left[-\frac{\left(1-\langle\cos \theta\rangle_{\theta}\right)}{T} t\right]-1\right) .
\end{aligned}
$$

Comparing with Eq. (21) we observe that the correlation time $\tau_{r}$ and the average flight time $T$ are related by

$$
\tau_{r}=\frac{T}{1-\langle\cos \theta\rangle_{\theta}}
$$

The average flight time $T$ can also be obtained from the definition of the Kuhn length as 40141

$$
b \equiv \lim _{L \rightarrow \infty} \frac{\left\langle r^{2}(t)\right\rangle}{L(t)}=L(T),
$$

where $L(t)=\sqrt{3} U_{e} t$ is the maximum length of the trajectory (see Appendix A]. Substitution of Eq. (25) and Eq. (26) into Eq. (27) yields

$$
b=\frac{2 U_{e} \tau_{r}}{\sqrt{3}}=\sqrt{3} U_{e} T
$$

and therefore

$$
\tau_{r}=\frac{3}{2} T, \quad\langle\cos \theta\rangle_{\theta}=\frac{1}{3} .
$$

From rotational diffusion ${ }^{42 / 43}$, the correlation time is related to the rotational diffusion coefficient $D_{r}$, in $d$ spatial dimensions, by $\tau_{r}=\left[(d-1) D_{r}\right]^{-1}$. Therefore for our micro-swimmer $T^{-1}=3 D_{r}$. The value of $D_{r}$ depends on the distribution of the torque that acts on the micro-swimmer, for example, reorientation due to thermal noise yields $D_{r}^{T}=k_{B} T / 8 \pi \mu R^{3}$ from the Stokes-Einstein relation 43 . In the next section we will calculate the value of $D_{r}$ explicitly, considering the variance of the torque $\mathbf{M}$ given by Eq. (2).

\subsubsection{Rotational diffusion coefficient}

We now calculate the rotational diffusion coefficient for the particle by considering the variance of its angular displacement. The orientation of the micro-swimmer at time $t$ can be obtained from its initial orientation by applying the rotation matrix $\mathbf{R}(t)=\exp [\Gamma(t)]$, where $\Gamma$ is a matrix such that for any vector $\mathbf{v}$ we have $\Gamma \mathbf{v}=\gamma \times \mathbf{v}$, with $\gamma$ the angle vector

$$
\gamma(t)=\int_{0}^{t} \omega(s) \mathrm{d} s .
$$

Given an orthonormal basis $\mathscr{B}=\left\{\mathbf{e}_{i}\right\}_{i=1}^{3}$, the components of $\gamma$ on $\mathscr{B}$ satisfy $\left\langle\cos \left|\gamma_{i}\right|\right\rangle=e^{-D_{r} t}$, which in the Gaussian limit (thermal noise ${ }^{42}$ ) reduces to $\operatorname{Var}\left[\gamma_{i}\right]=2 D_{r} t$, where $\operatorname{Var}[\cdot]$ denotes the variance. For large $N$ we may assume that $\gamma$ is spherically uniform, 
thus we define the rotational diffusion coefficient as

$$
6 D_{r} \equiv \lim _{t \rightarrow \infty} \frac{\left[\left\langle|\gamma|^{2}\right\rangle-|\langle\gamma\rangle|^{2}\right]}{t}
$$

Substituting Eq. (2) in Eq. (30) leads to

$$
\gamma=-\frac{f}{8 \pi \mu R^{2}} \int_{0}^{t} \sum_{i=1}^{N} \varepsilon_{i}(s)\left(\mathbf{e}_{r_{i}}(s) \times \mathbf{p}_{i}(s)\right) \mathrm{d} s \equiv \int_{0}^{t} \sum_{i=1}^{N} \varepsilon_{i}(s) \mathbf{c}_{i}(s),
$$

where Eq. (32) defines the vectors $\mathbf{c}_{i}(s)$. The variance of the angle vector for a fixed configuration of attached bacteria is then given by

$$
\begin{aligned}
\operatorname{Var}[\gamma] & =\int_{0}^{t} \int_{0}^{t} \sum_{i, j}^{N}\left[\left\langle\varepsilon_{i}\left(s_{1}\right) \varepsilon_{j}\left(s_{2}\right)\right\rangle_{\varepsilon}-\left(p_{R}^{s}\right)^{2}\right] \mathbf{c}_{i}\left(s_{1}\right) \cdot \mathbf{c}_{j}\left(s_{2}\right) \mathrm{d} s_{2} \mathrm{~d} s_{1} \\
& =\int_{0}^{t} \int_{0}^{t}\left[p_{R}^{s} p_{R R}\left(\left|s_{2}-s_{1}\right|\right)-\left(p_{R}^{s}\right)^{2}\right] \sum_{i=1}^{N} \mathbf{c}_{i}\left(s_{1}\right) \cdot \mathbf{c}_{i}\left(s_{2}\right) \mathrm{d} s_{2} \mathrm{~d} s_{1} \\
& =\int_{0}^{t} \int_{0}^{t} p_{R}^{s} p_{T}^{s} e^{-\left(\lambda_{R}+\lambda_{T}\right)\left|s_{2}-s_{1}\right|} \sum_{i=1}^{N} \mathbf{c}_{i}\left(s_{1}\right) \cdot \mathbf{c}_{i}\left(s_{2}\right) \mathrm{d} s_{2} \mathrm{~d} s_{1} .
\end{aligned}
$$

For large $N$ we may replace the sum by $N$ times the average over configurations

$$
\sum_{i=1}^{N} \mathbf{c}_{i}\left(s_{1}\right) \cdot \mathbf{c}_{i}\left(s_{2}\right) \simeq N\left(\frac{f}{8 \pi \mu R^{2}}\right)^{2}\left\langle\left(\mathbf{e}_{r_{i}} \times \mathbf{p}_{i}\right)_{s_{1}} \cdot\left(\mathbf{e}_{r_{i}} \times \mathbf{p}_{i}\right)_{s_{2}}\right\rangle_{\mathbf{c}_{i}},
$$

where the subscript $\mathbf{c}_{i}$ denotes that the average is taken over the distributions of $\mathbf{e}_{r_{i}}$ and $\mathbf{p}_{i}$, which is equivalent to the average over the angles $\vartheta_{i}, \varphi_{i}, \alpha_{i}$ and $\beta_{i}$. As the positions and orientations of the bacteria are identically and independently distributed, the correlation function on the right hand side of Eq. (34) does not depend on $i$, hence

$$
\begin{gathered}
\left\langle\left(\mathbf{e}_{r} \times \mathbf{p}\right)_{s_{1}} \cdot\left(\mathbf{e}_{r} \times \mathbf{p}\right)_{s_{2}}\right\rangle_{\mathbf{c}}=N \sum_{i, j}\left\langle\left(\mathbf{e}_{r_{i}} \times \mathbf{p}_{i}\right)_{s_{1}} \cdot\left(\mathbf{e}_{r_{j}} \times \mathbf{p}_{j}\right)_{s_{2}}\right\rangle_{\mathbf{c}} \\
=N\left\langle\mathbf{b}\left(s_{1}\right) \cdot \mathbf{b}\left(s_{2}\right)\right\rangle_{\mathbf{c}}=N\left\langle|\mathbf{b}|^{2}\right\rangle_{\mathbf{c}}\left\langle\hat{\mathbf{b}}\left(s_{1}\right) \cdot \hat{\mathbf{b}}\left(s_{2}\right)\right\rangle_{\mathbf{c}}
\end{gathered}
$$

Remembering that $\mathbf{b}$ defines the direction of motion in our coarsegrained model, we have

$$
\left\langle\hat{\mathbf{b}}\left(s_{1}\right) \cdot \hat{\mathbf{b}}\left(s_{2}\right)\right\rangle_{\mathbf{c}}=\frac{1}{U_{e}^{2}} \mathscr{C}\left(\left|s_{2}-s_{1}\right|\right)=e^{-2 D_{r}\left|s_{2}-s_{1}\right|},
$$

from Eq. (24). On the other hand, since $\alpha_{i}$ is the angle between $\mathbf{e}_{r_{i}}$ and $\mathbf{p}_{i}$ and the bacterial distributions are independent and identical, then $\left\langle|\mathbf{b}|^{2}\right\rangle_{\mathbf{c}}=N\left\langle\sin ^{2} \alpha\right\rangle_{\alpha}$. Therefore

$$
\sum_{i=1}^{N} \mathbf{c}_{i}\left(s_{1}\right) \cdot \mathbf{c}_{i}\left(s_{2}\right) \simeq N\left(\frac{f}{8 \pi \mu R^{2}}\right)^{2}\left\langle\sin ^{2} \alpha\right\rangle_{\alpha} e^{-2 D_{r}\left|s_{2}-s_{1}\right|}
$$

Note that, in doing this approximation, we are ignoring the periodic component of the correlation function of the vectors $\mathbf{c}_{i}$. As it can be seen in Fig. 7 this decays on a time scale shorter than $\tau_{r}$ and, since we are interested on the diffusive behaviour of the micro-swimmer, we can ignore it* A substitution of Eq. (37) into Eq. (32) yields

$$
\begin{aligned}
\operatorname{Var}[\gamma] & =\frac{2 N f^{2}\left\langle\sin ^{2} \alpha\right\rangle_{\alpha}}{\left(8 \pi \mu R^{2}\right)^{2}} \int_{0}^{t} \int_{s_{1}}^{t} p_{R}^{s} p_{T}^{s} e^{-\left(\lambda_{R}+\lambda_{T}+2 D_{r}\right)\left(s_{2}-s_{1}\right)} \mathrm{d} s_{2} \mathrm{~d} s_{1} \\
& =\frac{2 N f^{2}\left\langle\sin ^{2} \alpha\right\rangle_{\alpha}}{\left(8 \pi \mu R^{2}\right)^{2}} p_{R}^{s} p_{T}^{s}\left(\frac{t}{\lambda_{R}+\lambda_{T}+2 D_{r}}\right) \\
& +\frac{2 N f^{2}\left\langle\sin ^{2} \alpha\right\rangle_{\alpha}}{\left(8 \pi \mu R^{2}\right)^{2}} p_{R}^{s} p_{T}^{s}\left(\frac{e^{-\left(\lambda_{R}+\lambda_{T}+2 D_{r}\right) t}-1}{\left(\lambda_{R}+\lambda_{T}+2 D_{r}\right)^{2}}\right)
\end{aligned}
$$

We expect the rotational diffusion time scale to be larger than the running and tumbling times, i.e. to be in the limit $D_{r} \ll\left(\lambda_{R}+\lambda_{T}\right)$, which will be verified later. In this limit, Eq. (38) simplifies to

$$
D_{r}=\left(\frac{f}{8 \pi \mu R^{2}}\right)^{2} \frac{N\left\langle\sin ^{2} \alpha\right\rangle_{0} \lambda_{R} \lambda_{T}}{3\left(\lambda_{R}+\lambda_{T}\right)^{3}}+\mathscr{O}\left(\frac{2 D_{r}}{\left(\lambda_{R}+\lambda_{T}\right)^{4}}\right) .
$$

We note that $D_{r}$ vanishes for $\alpha_{i}=0$, and indeed if all the bacteria are oriented in the radial direction they produce no momentarm torque from their propulsive force and there is no change in the orientation of the particle. To treat this case one must include the effect of the reaction torques, the calculations are however more complicated since the direction of $\mathbf{M}_{T}$ varies in time with respect to the direction of $\mathbf{M}_{R}$. On the other hand, the effects of thermal diffusion (which had been neglected) can be easily included by adding $6 D t$ to the MSD and by replacing $D_{r}$ by $D_{r}+D_{r}^{T}$ where $D=k_{B} T / 6 \pi \mu R$ and $D_{r}^{T}=k_{B} T / 8 \pi \mu R^{3}$ are the thermal translational and rotational diffusion coefficients respectively.

In the particular case of a micro-swimmer immersed in water we have $\mu \simeq 10^{-3} \mathrm{pNs} / \mu \mathrm{m}^{2}$ and with the parameters $f \simeq 5 \times 10^{-1} \mathrm{pN}, \lambda_{R} \simeq 10 \mathrm{~s}^{-1}, \lambda_{T} \simeq 1 \mathrm{~s}^{-1}$ and $\rho \simeq 10^{-1} \mu \mathrm{m}^{-2}$, we obtain

$$
D_{r} \simeq\left(2 \mu \mathrm{m}^{2} \mathrm{~s}^{-1}\right)\left\langle\sin ^{2} \alpha\right\rangle_{\alpha} R^{-2} .
$$

Therefore Eq. (39) is valid as long as we have $\left(0.2 \mu \mathrm{m}^{2}\right)\left\langle\sin ^{2} \alpha\right\rangle_{\alpha} R^{-2} \ll 1$. For a uniform distribution of angle of attachment in the range $\left[\alpha_{\min }, \alpha_{\max }\right],\left\langle\sin ^{2} \alpha\right\rangle_{\alpha} \simeq 0.71$, hence the condition $D_{r} \ll\left(\lambda_{R}+\lambda_{T}\right)$ is accomplished provided that the particle is sufficiently large, $R \gtrsim 3 \mu \mathrm{m}$. We now proceed to calculate the micro-swimmers effective speed.

\subsubsection{Effective speed}

We define the effective speed as the ensemble average projection of the velocity along the angular velocity, that is

$$
U_{e}^{2} \equiv\left\langle\frac{\left(\langle\mathbf{V}\rangle_{\varepsilon} \cdot\langle\omega\rangle_{\varepsilon}\right)^{2}}{\left|\langle\omega\rangle_{\varepsilon}\right|^{2}}\right\rangle_{\mathbf{p}}=\left(\frac{f p_{R}^{s}}{6 \pi \mu R}\right)^{2}\left\langle(\mathbf{a} \cdot \hat{\mathbf{b}})^{2}\right\rangle_{\mathbf{p}}
$$

where $\langle\cdot\rangle_{\mathbf{p}}$ denotes the average over configurations. For a large number of bacteria $N$, we may assume that the vector $\mathbf{a}$ is uniformly distributed on the sphere and therefore

* The torque $\mathbf{M}$ fluctuates around its mean $\langle\mathbf{M}\rangle_{\varepsilon} \propto \mathbf{b}$ on a time-scale in the order of $\lambda_{T}^{-1}$ which as seen below is much smaller than $\tau_{r}$. 


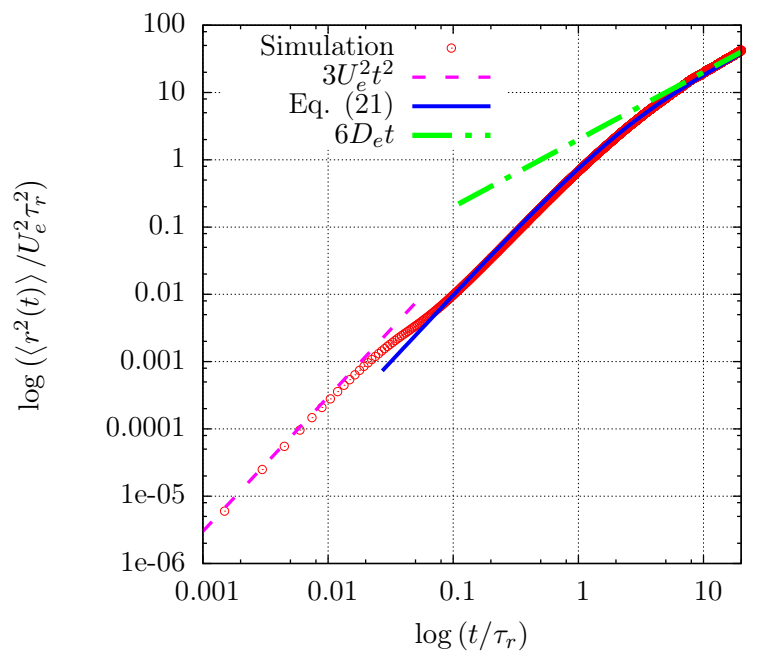

Fig. 6 Mean squared displacement of bacteria-driven swimmers as a function of time, for fixed radius $R=10 \mu \mathrm{m}$ and density of cells $\rho=$ $1 / 12 \mu \mathrm{m}^{-2}$. The parameters $f, \lambda_{T}$ and $\lambda_{R}$ are the same as in Fig. 4 Circles: mean values for $10^{4}$ different numerical realisations. Solid blue line; theory from Eq. 21]. Dashed dotted green line: asymptotic diffusive behaviour $\left\langle r^{2}\right\rangle=6 D_{e} t$ and $D_{e}$ given by Eq. (43). Dashed magenta line: instantaneous ballistic evolution of the MSD, $\left\langle r^{2}\right\rangle=3 U_{e}^{2} t^{2}$. Note that time is measured in units of $\tau_{r} \simeq 68 \mathrm{~s}$.

$\langle\mathbf{a} \cdot \hat{\mathbf{b}}\rangle_{\mathbf{p}}=\left\langle|\mathbf{a}|^{2}\right\rangle_{\mathbf{p}} / 3$. Substitution in Eq. 41) yields

$$
U_{e}^{2}=\left(\frac{f p_{R}^{s}}{6 \pi \mu R}\right)^{2} \frac{1}{3} \sum_{i, j}^{N}\left\langle\mathbf{p}_{i} \cdot \mathbf{p}_{j}\right\rangle_{\mathbf{p}}=\frac{N}{3}\left(\frac{f}{6 \pi \mu R} \frac{\lambda_{R}}{\lambda_{R}+\lambda_{T}}\right)^{2}
$$

where we have assumed that the bacteria behave independently of each other, that is $\left\langle\mathbf{p}_{i} \cdot \mathbf{p}_{j}\right\rangle=\delta_{i, j}$. Notice that $p_{R}^{s}$ is the fraction of time that the bacteria exert a force on the sphere, hence the effective speed is simply the average swimming speed of each bacterium $v_{0} \equiv f p_{R}^{s} /(6 \pi \mu R)$ multiplied by the average number of bacteria pushing along the axis of the helical path $\sqrt{N / 3}$. Notably, for a fixed density of attached bacteria, $U_{e}$ is independent of the size of the micro-swimmer as $N \sim R^{2}$, this is in agreement with the numerical results of Arabagi et al.14. On the other hand, $U_{e}$ is proportional to the square root of the number of bacteria, which is in agreement with the experimental results of Behkam et al.16.

\subsubsection{Long-time diffusion of bacteria-driven micro- swimmers}

We now use the results above to obtain an analytical expression for the effective diffusion coefficient, $D_{e}$, defined by Eq. (22). Substitution of Eqs. (39) and (42) into Eq. (22) with $\tau_{r}^{-1}=2 D_{r}$ leads to

$$
6 D_{e}=\frac{16}{9} \frac{R^{2} \lambda_{R}\left(\lambda_{R}+\lambda_{T}\right)}{\lambda_{T}\left\langle\sin ^{2} \alpha\right\rangle_{\alpha}}
$$

Our result makes two predictions with important consequences for experiments: (i) for a setup with a uniform density of surfaceattached bacteria, we predict that the long-time diffusion coefficient increases with the square of the size of the particle; (ii) the diffusion constant is, perhaps surprisingly, independent of the value of the propulsive force exerted by each bacterium. Recall however that diffusion is obtained in the "long-time" limit, which

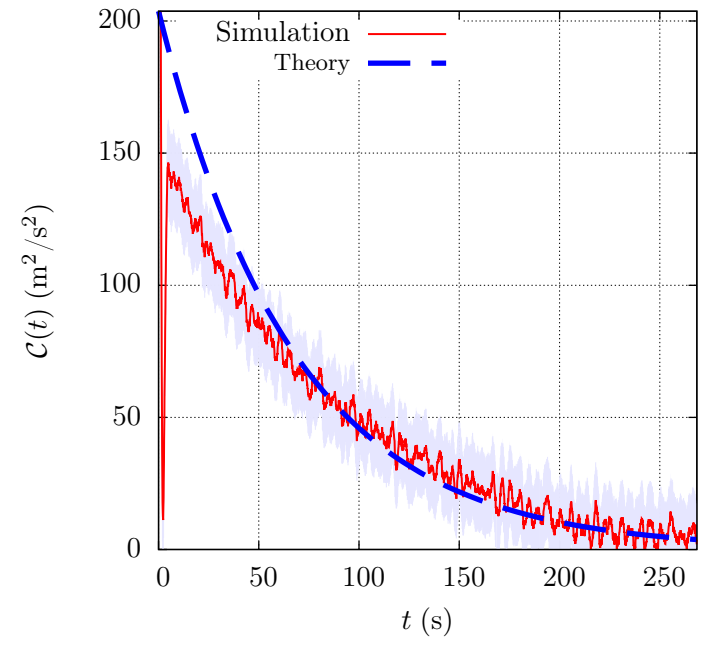

Fig. 7 Velocity correlation function of a bacteria-driven swimmer of radius $R=10 \mu \mathrm{m}$ and density of bacteria $\rho=1 / 12 \mu \mathrm{m}^{-2}$. The parameters $f, \lambda_{T}$ and $\lambda_{R}$ are the same as in Fig. 4 The solid line shows the average over $10^{4}$ realisations and the shaded region the standard deviation. The dashed line is the theoretical prediction from Eq. (24).

is defined as $t \gg D_{r}^{-1} \sim f^{-2}$ and therefore a variation in the value of $f$ changes this limit. Note that when $\alpha_{i}=\pi / 2$ for all $i$, we obtain a maximum value for the rotational diffusion and therefore a minimum value of the linear diffusion. In contrast, when $\alpha_{i}=0$ for all swimmers we obtain a singular expression for the diffusion coefficient, due to the fact that there is no reorientation as discussed in Sec. 3.2.1. This limit is treated in Appendix A

We next validate our analytical results against numerical simulations. In Fig. 6 we show a log-log plot of the MSD as a function of time for a micro-swimmer of radius $R=10 \mu \mathrm{m}$, with density of attached bacteria $\rho=1 / 12 \mu \mathrm{m}^{-2}$, a propulsive force $f=0.48 \mathrm{pN}$ and transition rates $\lambda_{T}^{-1}=0.9 \mathrm{~s}$ and $\lambda_{R}^{-1}=0.1 \mathrm{~s}$. Time is measured in units of $\tau_{r} \simeq 68 \mathrm{~s}$. At very short times $t \lesssim \tau_{r} / 100 \sim \lambda_{T}^{-1}=0.9 \mathrm{~s}$, the force and torque remain constant and therefore the MSD is simply the instantaneous mean squared velocity multiplied by the time squared, that is $\left\langle r^{2}\right\rangle=3 U_{e}^{2} t^{2}$ (dashed magenta line). The solid blue line is Eq. (21) and, for times-scales shorter than $\tau_{r}$, is ballistic with a MSD approximately $\left\langle r^{2}\right\rangle \simeq U_{e}^{2} t^{2}$. On the other hand, for time-scales larger than $\tau_{r}$, the MSD approaches the asymptotic limit $\left\langle r^{2}\right\rangle \simeq 6 D_{e} t$ represented by the dashed dotted green line, with $D_{e}$ given by Eq. (43).

On Fig. 7 we illustrate the exponential decay of the velocity correlation function (solid red line) for the same micro-swimmer. The dashed line is the theoretical prediction from Eq. (24) with $D_{r}=\left(2 \tau_{r}^{-1}\right)$ and $U_{e}$ given by Eqs. (39) and (42) respectively. The shaded region is the (numerical) standard deviation. The coarsegrained model captures accurately the late time behaviour of the computational results. Finally, Fig. 8 shows the dependence of $D_{e}$ with the bead radius $R$, for a micro-swimmer with the same set of parameters $f, \rho, \lambda_{R}$ and $\lambda_{T}$. The red circles and error bars represent the numerical mean and the standard deviation of $D_{e}$, which where obtained from a linear fit to the MSD in the regime $t>3 \tau_{r}$. The dashed blue line represents our theoretical prediction Eq. (43), which agrees very well with the numerical results. The 


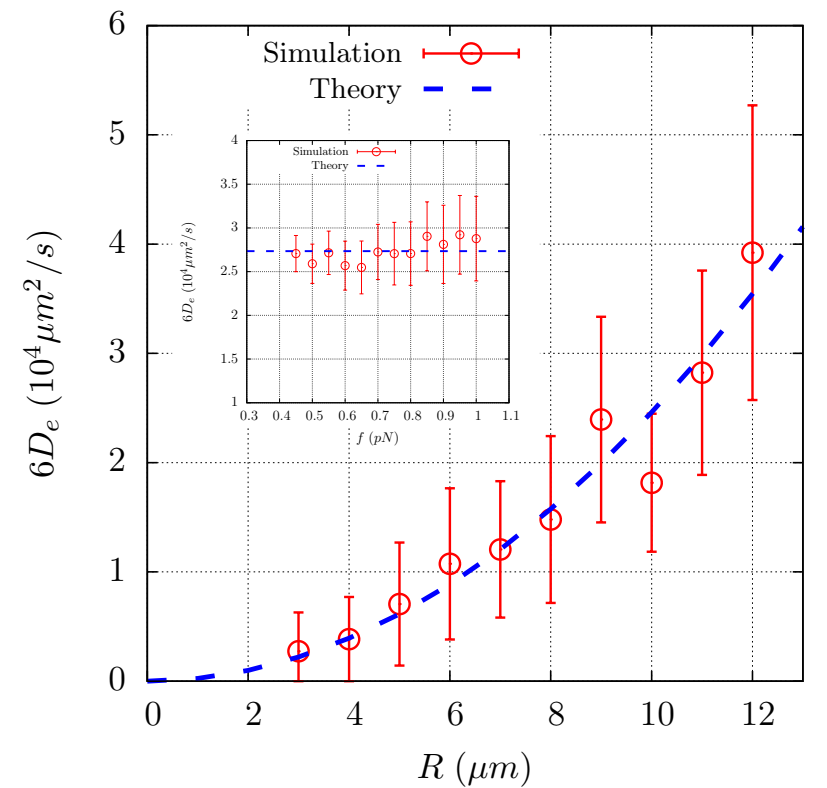

Fig. 8 Effective diffusion coefficient of bacteria-driven swimmer as a function of the bead radius, $R$, for fixed density of cells $\rho=1 / 12 \mu \mathrm{m}^{-2}$. The parameters $f, \lambda_{T}$ and $\lambda_{R}$ are the same as in Fig. 4 Inset shows $D_{e}$ as a function of the propulsive force $f$, for a micro-swimmer of radius $R=10 /, \mu \mathrm{m}$ and the same set of parameters. Mean values are shown in circles, error bars represent one standard deviation above and below the mean and the dashed line is the theoretical prediction of Eq. (43).

inset in Fig. 8 confirms that $D_{e}$ is independent of the propulsive force of the individual bacteria, $f$.

\subsection{Chemotaxis of bacteria-driven micro-swimmers}

In the previous sections, we analysed the motion of the microswimmers in chemically-homogeneous environments. Here we consider the case where instead the surrounding environment has a (weak) gradient of a solute to which the cells respond chemotactically. The question then emerges, whether the chemotaxis of individual cells translates to chemotaxis of the particle to which they are attached? The answer is not obvious a priori since bacteria in diametrically opposite positions on the particle can modify its motion in a symmetric fashion and as a result the chemotactic response can be vanishing. Experiments show however that the micro-swimmers perform indeed chemotaxis $13 \mid 15$. Here we rationalise this result using the coarse grained model developed in the last section and we give an analytical expression for the chemotactic drift, which is validated against numerical simulations.

\subsubsection{Chemotactic drift speed}

As illustrated in the inset of Fig. 9, when the tumbling rate varies according to Eq. (12), the trajectories are biased in the direction of increasing concentration (positive $z$ ) and the drift increases with the magnitude of the chemical gradient. This result can be rationalised observing the dependence of the average displacement on the magnitude of the chemical gradient. Using the coarse-grained model developed in Sec. 3.2 and following the classical de Gennes analysis for a single cell 354446 , we will compute the chemotactic drift speed $v_{d}$. The drift speed is obtained by averaging the mean distance travelled in a particular run, over the possible directions that the run can take and dividing the result by the average flight time. As the tumbling rate does not change when the bead moves in the plane perpendicular to $\nabla c$, the average displacement is non vanishing only in the direction of the gradient (i.e. z). Hence the drift velocity is

$$
v_{d}=\frac{\langle z\rangle}{T_{0}}=\frac{1}{T_{0}}\left\langle\int_{0}^{\infty} z(t) Q(t) \mathrm{d} t\right\rangle_{\hat{\mathbf{u}}},
$$

where $Q(t) \mathrm{d} t$ is the probability of tumbling between $t$ and $t+\mathrm{d} t$ and the subscript $\hat{\mathbf{u}}$ denotes that averages are taken over the directions of the velocity. Let us denote by $P(t)$ the probability of not stopping within $t$ seconds. By the same argument we used to compute $\mathscr{C}(t)$ in Sec. 3.2 we know that $P(t)$ is exponentially decaying and decays with rate $1 / T$, i.e.

$$
P(t)=\exp \left[-\int_{0}^{t} \frac{1}{T\left(t^{\prime}\right)} \mathrm{d} t^{\prime}\right]
$$

and $P(0)=1$. The probability of stopping after $t$ seconds is therefore given by

$$
1-P(t)=\int_{0}^{t}-\frac{d P\left(t^{\prime}\right)}{\mathrm{d} t^{\prime}} \mathrm{d} t^{\prime}=\int_{0}^{t} Q\left(t^{\prime}\right) \mathrm{d} t^{\prime} .
$$

Substitution of Eqs. (45) and (46) into Eq. (44) and using integration by parts gives the result

$$
v_{d}=\frac{1}{T_{0}}\left\langle\int_{0}^{\infty} w(t) \exp \left[-\int_{0}^{t} \frac{1}{T\left(t^{\prime}\right)} \mathrm{d} t^{\prime}\right] \mathrm{d} t\right\rangle_{\hat{\mathbf{u}}},
$$

where $w=\mathrm{d} z / \mathrm{d} t$ is the velocity of the particle in the direction of the gradient. Remembering that $T^{-1}=3 D_{r}$ from Eq. (29), and using Eq. (12) we have

$$
\frac{1}{T(t)}=\frac{1}{T_{0}}\left(1-\int_{-\infty}^{t} K\left(t-t^{\prime}\right) c\left(t^{\prime}\right) \mathrm{d} t^{\prime}\right)+\mathscr{O}\left(|\nabla c| \frac{\lambda_{0}}{\lambda_{R}}\right),
$$

this means that for shallow gradients, the tumbling rate for the micro-swimmer decreases in the same fashion as the tumbling rate for an individual bacterium, as long as $\lambda_{0} \ll \lambda_{R}$. We use Eq. (48) to expand $P(t)$ up to linear order in the gradient as follows

$$
P(t) \simeq e^{-t / T_{0}}+e^{-t / T_{0}} \frac{|\nabla c|}{T_{0}} \int_{0}^{t} \int_{-\infty}^{t^{\prime}} K\left(t^{\prime}-t^{\prime \prime}\right) z\left(t^{\prime \prime}\right) \mathrm{d} t^{\prime \prime} \mathrm{d} t^{\prime},
$$

where we have ignored the constant $c_{0}$ which gives terms that do not contribute to the drift, when we consider adaptive kernels with $\int K(t) \mathrm{d} t=0$. Inserting Eq. (49) in Eq. (47) and expressing $K(t)$ as a superposition of impulses we obtain the drift velocity as

$$
v_{d}=\int_{0}^{\infty} \int_{0}^{t} \int_{0}^{\infty} \int_{0}^{t^{\prime}-s} \frac{|\nabla c| e^{-t / T_{0}}}{T_{0}^{2}} K(s)\left\langle w(t) w\left(s^{\prime}\right)\right\rangle_{\hat{\mathbf{u}}} \mathrm{d} s^{\prime} \mathrm{d} s \mathrm{~d} t^{\prime} \mathrm{d} t .
$$

Here we have assumed that the distribution of $\hat{\mathbf{u}}$ is still uniform at first order in $|\nabla c|$, hence $\langle w\rangle_{\hat{\mathbf{u}}}=0$ and the velocity correlation along $z$ is given by

$$
\left\langle w(t) w\left(s^{\prime}\right)\right\rangle_{\hat{\mathbf{u}}}=\frac{U_{e}^{2}}{3}\left\langle\hat{\mathbf{u}}(t) \cdot \hat{\mathbf{u}}\left(s^{\prime}\right)\right\rangle_{\hat{\mathbf{u}}}=\frac{U_{e}^{2}}{3} e^{-2 D_{r}\left|t-s^{\prime}\right|},
$$




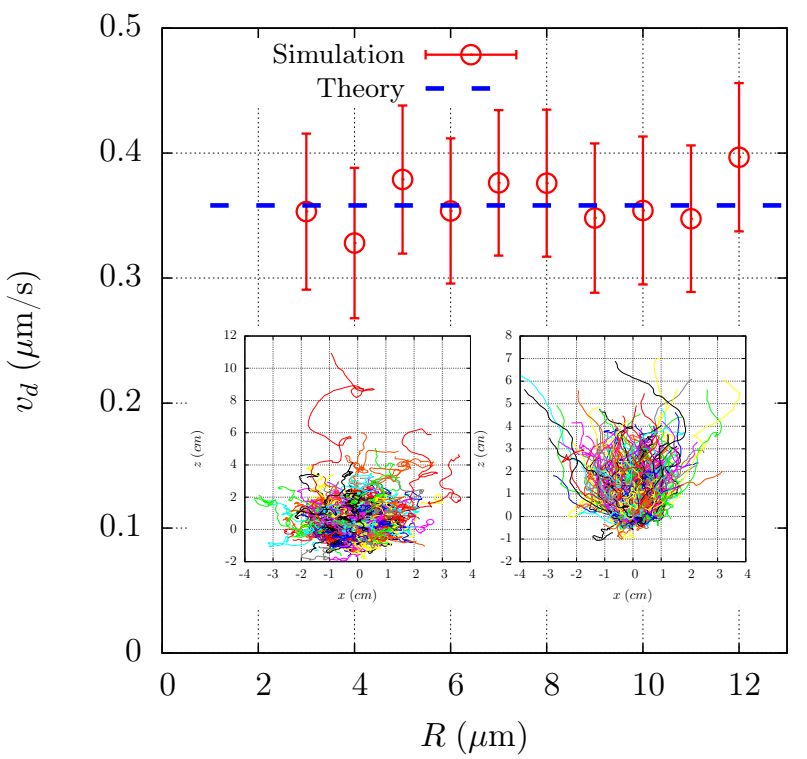

Fig. 9 Drift speed of bacteria-driven micro-swimmers as a function of the bead radius, $R$, for fixed cell density. The parameters $\rho, f$ and $\lambda_{R}$ are the same as in Fig. $4 \lambda_{0}^{-1}=0.9 \mathrm{~s}$ and $\kappa|\nabla c|=0.005 \mu \mathrm{m}^{-1}$. Symbols show mean values from the simulations, error bars represent the standard deviation and the dashed line is the theoretical prediction in Eq. (54). The inset shows the biased trajectories in the direction of the chemical gradient for $\kappa|\nabla c|=0.01$ (left) and $\kappa|\nabla c|=0.05$ (right) with each figure showing $10^{3}$ trajectories for a micro-swimmer of radius $R=10 \mu \mathrm{m}$ and the same set of parameters. In all cases, the simulation time is $t=50 /\left(3 D_{r}\right) \simeq 2240 \mathrm{~s}$, where $D_{r}$ is given by Eq. (39).

where we have used the fact that $\hat{\mathbf{u}}$ is spherically symmetric. Substitution of Eq. (51) into Eq. (50) yields the drift as

$$
\begin{aligned}
v_{d} & =\frac{|\nabla c| U_{e}^{2}}{6 T_{0}^{2} D_{r}} \int_{0}^{\infty} e^{-\left(1 / T_{0}+2 D_{r}\right) t} \int_{0}^{t} \int_{0}^{\infty} K(s)\left(e^{-2 D_{r}\left(s-t^{\prime}\right)}-1\right) \mathrm{d} s \mathrm{~d} t^{\prime} \mathrm{d} t \\
& =\frac{|\nabla c| U_{e}^{2}}{6 T_{0}^{2} D_{r}} \int_{0}^{\infty} K(s) e^{-2 D_{r} s} \mathrm{~d} s \int_{0}^{\infty} e^{-\left(1 / T_{0}+2 D_{r}\right) t} \int_{0}^{t} e^{2 D_{r} t^{\prime}} \mathrm{d} t^{\prime} \mathrm{d} t \\
& =\frac{|\nabla c| U_{e}^{2}}{12 T_{0}^{2} D_{r}^{2}} \int_{0}^{\infty} K(s) e^{-2 D_{r} s} \mathrm{~d} s \int_{0}^{\infty} e^{-t / T_{0}}-e^{-\left(1 / T_{0}+2 D_{r}\right) t} \mathrm{~d} t \\
& =\frac{|\nabla c| U_{e}^{2}}{2 D_{r}\left(3+6 D_{r} T_{0}\right)} \int_{0}^{\infty} K(s) e^{-2 D_{r} s} \mathrm{~d} s .
\end{aligned}
$$

It is known that $K(t)$ vanishes for $t>4 s \sqrt{26}$. Furthermore, $D_{r}$ decreases quadratically with increasing $R$, therefore for sufficiently large radius of the particle we have $2 D_{r} s \ll 1$ for all relevant values of $s$ (for example, when $R=10 \mu \mathrm{m}$ and $\rho=1 / 12 \mu \mathrm{m}^{-2}$, $2 D_{r} \simeq 0.015 \mathrm{~s}^{-1}$ ) and we may then expand the exponential in the last line of Eq. (52) to first order in the rotational diffusion coefficient. This leads to the general prediction for drift velocity

$$
v_{d}=-\frac{|\nabla c| U_{e}^{2}}{3+6 D_{r} T_{0}} \int_{0}^{\infty} K(s) s \mathrm{~d} s=-\frac{|\nabla c| U_{e}^{2}}{5} \int_{0}^{\infty} K(s) s \mathrm{~d} s .
$$

where we have used Eqs. (29) which leads to $T_{0}^{-1}=3 D_{r}$. Our prediction for $v_{d}$ is seen to be independent of the radius of the particle and is linear in the concentration gradient.

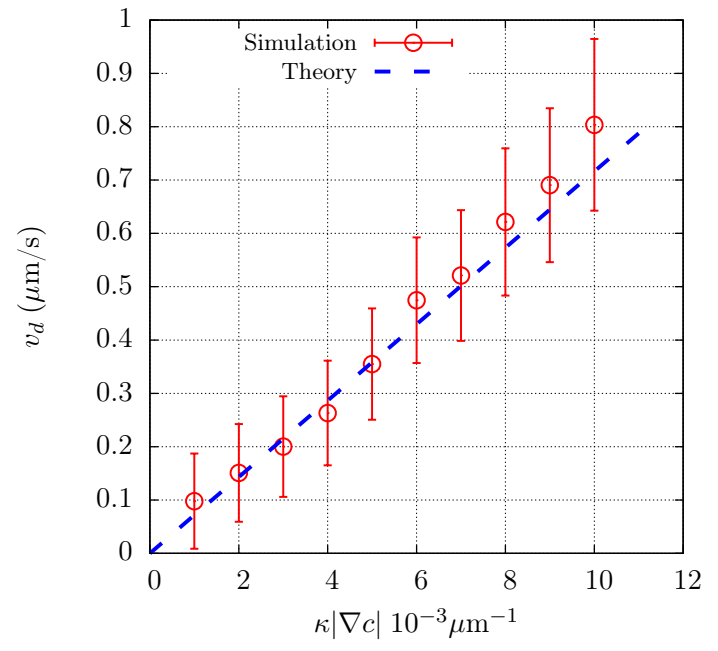

Fig. 10 Drift speed of bacteria-driven micro-swimmers as a function of $\kappa|\nabla c|$ for a particle of fixed radius $R=10 \mu \mathrm{m}$, The parameters $\rho, f$ and $\lambda_{R}$ are the same as in Fig. 4 The remaining parameters are the same as in Fig. 9 Symbols show mean values from the simulations, error bars represent the standard deviation and the dashed line is our prediction from Eq. (54).

For the particular choice of $K(t)=\kappa\left[\delta\left(t-t_{1}\right)-\delta\left(t-t_{2}\right)\right]$ used in our computations, we obtain

$$
v_{d}=\frac{\kappa|\nabla c| U_{e}^{2}\left(t_{2}-t_{1}\right)}{5} .
$$

These results are validated against our simulations in Figs. 9 and 10. In Fig. 9 we first plot $v_{d}$ as a function of $R$ for fixed $\kappa|\nabla c|$, $t_{1}=1 \mathrm{~s}, t_{2}=3 \mathrm{~s}, \lambda_{0}^{-1}=0.9 \mathrm{~s}$ and the same set of parameters $f, \rho$ and $\lambda_{R}$ as in the isotropic case. Correspondingly, we plot in Fig. 10 the drift speed as a function of $\kappa|\nabla c|$ for fixed radius $R=10 \mu \mathrm{m}$ and the same set of parameters. Once again, the red circles and error bars represent the numerical values of the mean and standard deviation of $v_{d}$. These values were obtained from a linear fit to the average displacement in the regime $t>10 \tau_{r}$. The dashed blue lines represent the prediction of Eq. (54) and we can observe that the agreement with the numerical results is excellent.

\section{Discussion}

In this paper, we developed a coarse-grained model that allows to accurately predict the diffusive behaviour of bacteria-driven micro-swimmers, both in homogeneous environments and in the presence of (weak) chemical gradients. The analytical expressions for the effective diffusion coefficient $D_{e}$ (Eq. 43) and the chemotactic drift speed $v_{d}$ (Eq. 54) were validated against numerical simulations using the stochastic model presented in Section 2.1 with Eq. (12) for the chemotactic change of the tumbling rate of the bacteria. We found that the effective swimmer velocity, $U_{e}$, is independent of the swimmer size, but increases with the square root of the number of surface-attached bacteria $N$, when $N$ is large. On the other hand, the rotational diffusion coefficient, $D_{r}$, increases linearly with $N$ and is inversely proportional to the particle radius $R$, in contrast with the thermal rotational diffusion coefficient $D_{r}^{T}$ which decreases as $R^{-3}$. For shallow chemical gra- 
dients $\kappa|\nabla c| R \ll 1$, we found that the micro-swimmers respond chemotactically if we assume that each bacterium on its surface does, and that the resulting chemotactic drift speed is proportional to $|\nabla c|$ and independent of $R$, the latter being a consequence of the bacteria being constrained by the bead and each bacterium experiencing the same change in concentration.

The expression we obtained for the effective velocity in this paper can be directly compared with experimental results. Behkam et al. reported $U_{e}=14.8 \pm 1.1 \mu \mathrm{m} / \mathrm{s}$ for micro-swimmers of radius $R=10 \mu \mathrm{m}$ and densities of attachment between one bacterium per $12 \mu \mathrm{m}^{2}$ and $7 \mu \mathrm{m}^{2}$ with $f \simeq 0.48 \mathrm{pN}^{16}$. In comparison, our model predicts $U_{e} \simeq 12.99-17.42 \mu \mathrm{m} / \mathrm{s}$, in good agreement. The same study also reported that the swimming speed increases with the square root of the number of bacteria $N$, for $N \geq 10$, as predicted theoretically. The same scaling result was reported by Arabagi et al.14 and Zhuang et al.15! Furthermore, both of these studies reported swimming velocities independent of the particle radius in homogeneous environments as well as in the presence of a chemical gradient, again confirming our theoretical results. Unfortunately there are no past experimental measurements of the effective diffusion coefficient. In their numerical simulations however, Arabagi et al. ${ }^{14}$ reported the value $D_{e}=15185 \mu \mathrm{m}^{2} / \mathrm{s}$ for a bead of radius $R=20 \mu \mathrm{m}$ and $f \simeq 0.48 \mathrm{pN}$, in good agreement with Eq. (43) which predicts $D_{e} \simeq 15802 \mu \mathrm{m}^{2} / \mathrm{s}$. Regarding the chemotactic drift of particles with surface-attached bacteria, Zhuang et al. 13115 found that $v_{d}$ is proportional to the magnitude of the chemical gradient and that it is independent of the particle size, both of which are predicted by Eq. (54).

The analytical results in this paper will be useful for the practical design of bacteria-driven micro-swimmers. The model can be extended to include other steering strategies such as phototaxis, $\mathrm{pH}$-taxis and external electromagnetic fields 2029 . The assumption of a chemically permeable particle could also be relaxed, and the perturbation to the concentration field by the presence of the particle (and the cells) should be solved for. Another possible extension is the study of deformable random walkers, inspired by experiments with bacteria-driven micro-swimmers in which E. coli are attached to red blood cells 47 . One of the ultimate applications of bacteria-driven micro-swimmers is targeted drug delivery, which requires a suspension of these particles to move collectively. A mathematical description of the collective behaviour of multiple micro-swimmers is therefore needed and this requires an explicit consideration of their hydrodynamic interactions. The interactions depend critically on the distribution of attached bacteria on the surface of each particle, which can be controlled using micro-manipulation $\sqrt{48}$ and nanoprinting methods ${ }^{5 / 49}$. A theory including hydrodynamic interactions, taking clues from classical work on active fluids ${ }^{50151}$ will therefore allow to move bacteriadriven micro-swimmers closer to applications.

\section{Conflicts of interest}

There are no conflicts to declare.

\section{Acknowledgements}

This project has received funding from the European Research Council (ERC) under the European Union's Horizon 2020 re- search and innovation programme (grant agreement 682754 to $\mathrm{EL})$.

\section{A Singular case $\alpha_{i}=0$}

When all bacteria point in the radial direction, there is no applied torque and every vector $\mathbf{p}_{i}$ remains constant in time. In this case, the velocity correlation function is easily computed as follows

$$
\begin{aligned}
\langle\mathbf{V}(0) \cdot \mathbf{V}(t)\rangle & =\left(\frac{f}{6 \pi \mu R}\right)^{2} \sum_{i, j}\left\langle\varepsilon_{i}(0) \varepsilon_{j}(t)\right\rangle \mathbf{p}_{i} \cdot \mathbf{p}_{j} \\
& =N\left(\frac{f}{6 \pi \mu R}\right)^{2} p_{R} p_{R R}(t),
\end{aligned}
$$

where we have used the fact that the orientations and positions of bacteria are uncorrelated. Integrating twice, we obtain the MSD

$$
\begin{aligned}
\left\langle r^{2}(t)\right\rangle & =2 N\left(\frac{f p_{R}^{s}}{6 \pi \mu R}\right)^{2} \frac{t^{2}}{2} \\
& +2 N\left(\frac{f p_{R}^{s}}{6 \pi \mu R}\right)^{2} \int_{0}^{t} \int_{s_{1}}^{t}\left(\frac{\lambda_{T}}{\lambda_{R}} e^{-\left(\lambda_{R}+\lambda_{T}\right)\left(s_{2}-s_{1}\right)}\right) \mathrm{d} s_{2} \mathrm{~d} s_{1} \\
& =2 N\left(\frac{f p_{R}^{s}}{6 \pi \mu R}\right)^{2} \frac{t^{2}}{2} \\
& +2 N\left(\frac{f p_{R}^{s}}{6 \pi \mu R}\right)^{2} \frac{\lambda_{T}}{\lambda_{R}\left(\lambda_{T}+\lambda_{R}\right)}\left(t+\frac{e^{-\left(\lambda_{R}+\lambda_{T}\right) t}-1}{\left(\lambda_{T}+\lambda_{R}\right)}\right) .
\end{aligned}
$$

This is essentially ballistic and for $1 \ll t\left(\lambda_{T}+\lambda_{R}\right)$ we have $\left\langle r^{2}(t)\right\rangle \simeq 3 U_{e}^{2} t^{2} \equiv L^{2}(t)$ with $U_{e}$ given by Eq. (42).

This result might appear counter intuitive, as one would expect that a large number of uniformly distributed radial forces acting on a sphere should account for no net thrust. However, this is only true if the time autocorrelation of the forces decays to zero. As the autocorrelation of the epsilon variables decays to a finite value, $\left(p_{R}^{s}\right)^{2}$ from Eq. (11), so does the velocity correlation function, hence the ballistic motion. Tilting the forces away from the radial direction allows for reorientation of the sphere, reducing the probability of finding a given force pointing in the same direction at two different times accounting for a vanishing autocorrelation at large time-scales, hence the long-time diffusive behaviour for the case $\alpha_{i} \neq 0$. Notice that $L(t)$ is the maximum average length that a trajectory can have (with $\alpha_{i}$ not necessarily null). Indeed, the arc length of a helix is the same as that of a straight line traversed at the same speed, therefore $L(t)$ can be obtained by considering the square root of Eq. (41) in the limit when $\langle\mathbf{V}\rangle_{\varepsilon}$ is parallel to $\langle\omega\rangle_{\varepsilon}$ and multiplying the result by $t$, which is exactly $L(t)=\left\langle\left|\mathbf{V}_{\varepsilon}\right|^{2}\right\rangle^{1 / 2} t=\sqrt{3} U_{e} t$.

\section{Notes and references}

1 M. Sitti, Nature, 2009, 458, 1121.

2 S. Martel, Biomed. Microdevices, 2012, 14, 1033-1045.

3 H. C. Berg, E. coli in Motion, Springer New York, 2004.

4 Z. Hosseinidoust, B. Mostaghaci, O. Yasa, B.-W. Park, A. V. Singh and M. Sitti, Adv. Drug Deliver. Rev., 2016, 106, 27 44. 
5 R. W. Carlsen and M. Sitti, Small, 2014, 10, 3831-3851.

6 H. Ceylan, J. Giltinan, K. Kozielski and M. Sitti, Lab Chip, 2017, 17, 1705-1724.

7 A. Julius, M. Sakar, E. Steager, U. Cheang, M. Kim, V. Kumar and G. Pappas, 2009 IEEE International Conference on Robotics and Automation, 2009, pp. 1004-1009.

8 N. Darnton, L. Turner, K. Breuer and H. C. Berg, Biophys. J., 2003, 86, 1863-1870.

9 J. Katuri, X. Ma, M. M. Stanton and S. Sánchez, Accounts Chem. Res., 2017, 50, 2-11.

10 L. Schwarz, M. Medina-Sánchez and O. G. Schmidt, Appl. Phys. Rev., 2017, 4, 031301.

11 H. Wang and M. Pumera, Adv. Funct. Mater., 2018, 28, 1705421.

12 J. Bastos-Arrieta, A. Revilla-Guarinos, W. E. Uspal and J. Simmchen, Front. Robot. AI, 2018, 5, 97.

13 J. Zhuang and M. Sitti, Sci. Rep-UK, 2016, 6, 32135.

14 V. Arabagi, B. Behkam, E. Cheung and M. Sitti, J. Appl. Phys., 2011, 109, 114702.

15 J. Zhuang, B.-W. Park and M. Sitti, Adv. Sci., 2017, 4, 1700109.

16 B. Behkam and M. Sitti, Appl. Phys. Lett., 2008, 93, 223901.

17 O. Schauer, B. Mostaghaci, R. Colin, D. Hürtgen, D. Kraus, M. Sitti and V. Sourjik, Sci. Rep., 2018, 8, 9801.

18 S. Cho, S. J. Park, S. Y. Ko, J.-O. Park and S. Park, Biomed. Microdevices, 2012, 14, 1019-1025.

19 V. D. Nguyen, J.-W. Han, Y. J. Choi, S. Cho, S. Zheng, S. Y. Ko, J.-O. Park and S. Park, Sensor. Actuat. B-Chem., 2016, 224, $217-224$.

20 E. B. Steager, M. S. Sakar, D. H. Kim, V. Kumar, G. J. Pappas and M. J. Kim, J. Micromech. Microeng., 2011, 21, 035001.

21 B. Behkam and M. Sitti, Appl. Phys. Lett., 2007, 90, 023902.

22 M. M. Stanton, J. Simmchen, X. Ma, A. Miguel-López and S. Sánchez, Adv. Mater. Interfaces, 2015, 3, 1500505.

23 E. Lauga, Annu. Rev. Fluid Mech., 2016, 48, 105-130.

24 D. Kim, A. Liu, E. Diller and M. Sitti, Biomed. Microdevices, 2012, 14, 1009-1017.

25 S. M. Block, J. E. Segall and H. C. Berg, Cell, 1982, 31, 215226.

26 J. E. Segall, S. M. Block and H. C. Berg, P. Natl. Acad. Sci. USA, 1986, 83, 8987-8991.

27 M. J. Schnitzer, Phys. Rev. E, 1993, 48, 2553-2568.

28 S. J. Park, S.-H. Park, S. Cho, D.-M. Kim, Y. Lee, S. Y. Ko, Y. Hong, H. E. Choy, J.-J. Min, J.-O. Park and S. Park, Sci. Rep. UK, 2013, 3, 3394.
29 E. Steager, C.-B. Kim, J. Patel, S. Bith, C. Naik, L. Reber and M. J. Kim, Appl. Phys. Lett., 2007, 90, 263901.

30 R. W. Carlsen, M. R. Edwards, J. Zhuang, C. Pacoret and M. Sitti, Lab Chip, 2014, 14, 3850-3859.

31 M. R. Edwards, R. W. Carlsen and M. Sitti, Appl. Phys. Lett., 2013, 102, 143701.

32 J. C. Simo, N. Tarnow and M. Doblare, Int. J. Numer. Meth. Eng., 1995, 38, 1431-1473.

33 E. Zupan and M. Saje, Adv. Eng. Softw., 2011, 42, 723 - 733.

34 V. Sourjik and N. S. Wingreen, Curr. Opin. Cell Biol., 2012, 24, $262-268$.

35 P.-G. de Gennes, Eur. Biophys. J., 2004, 33, 691-693.

36 D. A. Clark and L. C. Grant, P. Natl. Acad. Sci. USA, 2005, 102, 9150-9155.

37 A. Celani and M. Vergassola, P. Natl. Acad. Sci. USA, 2010, 107, 1391-1396.

38 S. Chandrasekhar, Rev. Mod. Phys., 1943, 15, 1-89.

39 P. S. Lovely and F. Dahlquist, J. Theor. Biol., 1975, 50, 477 496.

40 M. Rubinstein and R. H. Colby, Polymer Physics (Chemistry), Oxford University Press, 2003.

41 H. Yamakawa, Helical Wormlike Chains in Polymer Solutions, Springer Berlin Heidelberg, 1997.

42 J. Saragosti, P. Silberzan and A. Buguin, PLOS ONE, 2012, 7, $1-6$.

43 M. Doi and H. See, Introduction to Polymer Physics, Oxford University Press, USA, 1996.

44 J. T. Locsei, J. Math. Biol., 2007, 55, 41-60.

45 J. Taktikos, H. Stark and V. Zaburdaev, PLoS ONE, 2013, 8, e81936.

46 E. V. Pankratova, A. I. Kalyakulina, M. I. Krivonosov, S. V. Denisov, K. M. Taute and V. Y. Zaburdaev, PLoS ONE, 2018, 13, e0190434.

47 Y. Alapan, O. Yasa, O. Schauer, J. Giltinan, A. F. Tabak, V. Sourjik and M. Sitti, Sci. Robotics, 2018, 3, eaar4423.

48 Á. Barroso, S. Landwerth, M. Woerdemann, C. Alpmann, T. Buscher, M. Becker, A. Studer and C. Denz, Biomed. Microdevices, 2015, 17, 26.

49 S. Rozhok, C.-F. Shen, P.-L. Littler, Z. Fan, C. Liu, C. Mirkin and R. Holz, Small, 2005, 1, 445-451.

50 M. C. Marchetti, J.-F. Joanny, S. Ramaswamy, T. B. Liverpool, J. Prost, M. Rao and R. A. Simha, Rev. Mod. Phys., 2013, 85, 1143.

51 F. Jülicher, S. W. Grill and G. Salbreux, Rep. Prog. Phys., 2018, 81, 076601. 